\title{
Obstructive sleep apnoea with co existent Kleine-Levine syndrome in a middle-aged man
}

\author{
Gopal Chawla1, Ram Niwas', Naveen Dutt' ${ }^{1}$, Mukesh Kumar Swamy² \\ ${ }^{1}$ Department of Pulmonary Medicine, All India Institute of Medical Sciences, Jodhpur; ${ }^{2}$ Department of Psychiatry, All \\ India Institute of Medical Sciences, Jodhpur, India
}

\begin{abstract}
Hypersomnia is a symptom which is pathognomonic of patients with Kleine-Levin syndrome (KLS), but the cause of this finding remains undefined. Given the pervasive association between obstructive sleep apnoea (OSA) and excessive daytime sleepiness, there exists the possibility that OSA might contribute to the sleepiness exhibited by these patients. Here we report a middle age man who had excessive daytime sleepiness and diagnosed as OSA after polysomnography. Prescribing of continuous positive airway pressure (CPAP) lead to unmasking of episodes of increased sleepiness and other behavioural features of KLS.
\end{abstract}

\section{Case Report}

A 36-year-old chef with BMI of 36.4 presented with complaints of excessive day time sleepiness, snoring and anxiety episodes on and off for past 1 year. He also mentioned about

Correspondence: Dr. Ram Niwas, Department of Pulmonary Medicine, All India Institute of Medical Sciences, Jodhpur, India. Mobile: +91.9999493179. E-mail: rniwasaiims@gmail.com

Conflict of interest: The Authors declare no conflict of interest.

Contributions: GC, ND, study concept; GC, RN, ND, study design, definition of intellectual contents; GC, RN, literature search; GC, RN data acquisition; GC, ND, MKS, manuscript drafting; GC, RN, ND, MKS, manuscript editing and review. All authors read and approved the final version to be published.

Key words: Sleep disordered breathing; Kleine-Levine syndrome; KLS; OSA.

Received for publication: 29 August 2019.

Accepted for publication: 30 October 2019.

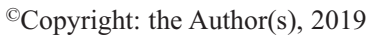

Licensee PAGEPress, Italy

Monaldi Archives for Chest Disease 2019; 89:1163

doi: 10.4081/monaldi.2019.1163

This article is distributed under the terms of the Creative Commons Attribution Noncommercial License (by-nc 4.0) which permits any noncommercial use, distribution, and reproduction in any medium, provided the original author(s) and source are credited. morning headache and feeling of tiredness even after sleep. Apart from this system he was involved in vehicular accident when he dozed off while driving and escaped with minor injuries. His Epworth sleepiness score was 20. Considering severity of the disease, he was planned for split night polysomnography. Apnoea and hypopnea index was equal to $34.8 / \mathrm{h}$, with basal saturation of $98 \%$ and minimum of $82 \%$. The positive pressure titration indicated an ideal pressure for apnoea and hypopnea control of $9 \mathrm{~cm} \mathrm{H}_{2} \mathrm{O}$ (Figure1).

At the next follow-up visit, the patient reported that his symptoms showed partial improvement with continuous positive airway pressure (CPAP). He followed CPAP up for 6 months, which compliance was ensured by verifying the data with memory card. Compliance was more that $85 \%$ but patient remain unsatisfied due to fact that his excessive sleepiness is gone for few days to return again with vengeance. Intrigued by kind of episodic nature of excessive sleepiness he was posted for detailed history session. On detailed history it was divulged that his symptoms are not continuous but episodic, which include excessive day time sleepiness and hypersomnia, along with excessive and compulsive eating with increase in sex drive, though all these three symptoms together were only present only in few episodes. He has these episodes for 3-4 days followed by symptom-free period for around 15 days. The history was confirmed with spouse and his brother. During this period, the patient sleeps for more than 12 hours and if woken up early gets irritated and sleeps again. Eating almost three times of what he takes usually. He continued to have heightened sexual urges. This hypersexual behaviour includes excessive masturbation along with forcing his partner which led to quarrels and abusive behaviour.

He underwent counselling session with psychologist and later Cognitive Behaviour Session with psychiatrist. MRI brain to rule out lesion was done, which came out to be normal. He underwent polysomnography during one of the episodes which showed increase in sleep time along with decrease in sleep and REM onset latency (Table 1). In view of persistence of episodes of hypersexuality, hypersomnolence and hyperphagia and contributory polysomnography, the patient was started on Lithium $300 \mathrm{mg}$ BD (serum lithium $0.5 \mathrm{mEq} / \mathrm{L}$ ) along with CPAP of $9 \mathrm{~cm} \mathrm{H}_{2} \mathrm{O}$, Patient showed symptomatic improvement with this treatment and is presently under follow-up for past 4 months.

\section{Discussion}

Eponym Kleine-Levin syndrome (KLS) was coined by Critchley and Hofmann when they described two young individual with these names in 1942. KLS is a rare disorder, world preva- 
lence being only $1-5 / 10^{6}$, mainly affecting adolescent males, in whom the principal features are intermittent hyper somnolence, hyperphagia, behavioural and cognitive disturbances like anxiety and in some cases hypersexuality. Each episode is of small varying duration from few days to 1-2 months and affected people are entirely asymptomatic between episodes [1,2].

New criteria changed the conventional Critchley triad and mentioned recurrent episodes of unexplained sleepiness lasting from a few days to 4 weeks, occurring at least annually, and may or may not be accompanied by impulsive and rapid consumption of large amounts of food (hyperphagia, binge eating), and/or hyper sexuality to diagnose KLS [2].

The disorder is more frequent among teenagers of the male gender and has an unpredictable course with remissions and recurrences, which can last for years. This disease can disappear as subtly as it appears (an average of 8-13 years duration) [3,4].

Our case was peculiar as the symptoms first appeared at age of 36 years, where the mean age was 15 years in systemic review of 186 cases, only 6 patients had late onset KLS, i.e. after 35 years [5]. Apart from being middle aged, the KLS symptoms were not initially present or if present were not bothersome but using of CPAP for obstructive sleep apnoea (OSA) led to unmasking of these symptoms. Also, the possibility is present that these symptoms appeared incidentally the first time.

It is quite astonishing that the diagnosis of a sleep disorder such as KLS cannot be reached by routine or even extended polysomnography (PSG). There are at times logistic difficulties in obtaining a PSG at the onset or peak of episodic sleepiness. Sometimes during an episode these patients exhibit behaviours that render them unable to withstand and or cooperate with PSG or multiple sleep latency test (MSLT). Reports of complete PSG in these patients are scarce. Those which were able to be performed and obtained only limited data is available, with sleep duration being the most common parameter reported $[6,7]$. As in our case reported time for sleep study during the episode was almost three times as compared to initial PSG.

It is even more surprising that the possibility of the presence of abnormal breathing patterns during this episodic hypersomnolence have not received greater attention. This may be because of absence of typical complaints associated with sleep-disordered breathing from these young and otherwise healthy patients, e.g., snoring or witnessed sleep apnoea. Breathing abnormalities during the hyper somnolent periods of KLS have only rarely been observed.

Discussing about co-existence apart from one case where a 14year adolescent diagnosed with KLS. PSG done 5 years later because of classical symptoms OSA revealed AHI of 30.8 [8]. Apart from this case, the only work was done by Lavie et al. where they showed that lean young patients of KLS had co-existent OSA [9], while in our patient presented with symptoms of OSA, the later episodic nature got unmasked.

Reason behind association of KLS and sleep apnoea is still not clear, it was postulated breathing abnormalities could be the result of 'central hypoexcitability,' bearing a similarity to the frequent observation of a central sleep apnoea following the transition from wakefulness to sleep [9]. Vigren et al. used the single-photon emission computed tomography (SPECT) to establish the fact that KLS-patients have hypoperfusion in the temporal or fronto-temporal regions, though only $48 \%$ showed this finding establishing the fact there is more to pathophysiology of KLS and its association with sleep disordered breathing [10].

Table 1. Comparison of polysomnography parameters during initial sleep study and later during symptomatic period, after 6 months.

Asymptomatic Symptomatic (episodic)

\begin{tabular}{lcc} 
Total sleep time (min) & 424 & 742 \\
Sleep efficiency (\%) & 84 & 86 \\
\hline Sleep latency (min) & 34 & 16 \\
REM latency(min) & 156 & 76 \\
\hline Stage 1 (\%) & 4 & 3 \\
Stage 2 (\%) & 73 & 70 \\
\hline Stage 3 (\%) & 15 & 12 \\
REM (\%) & 9 & 14 \\
\hline
\end{tabular}

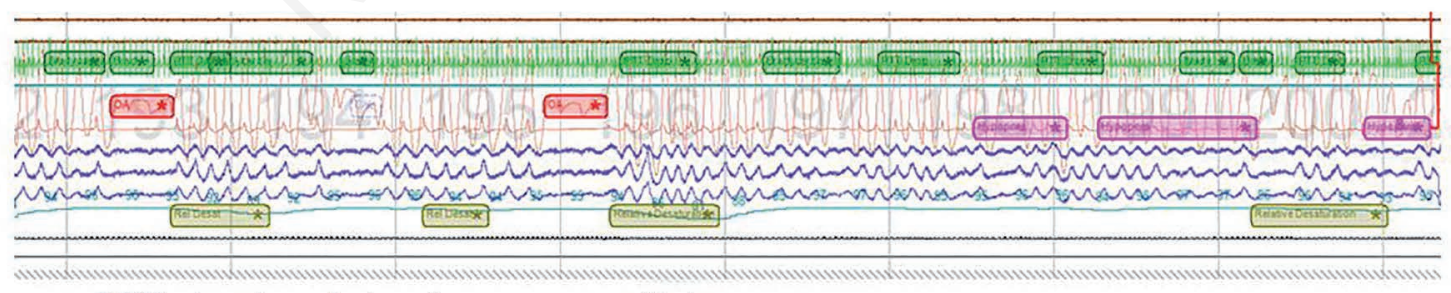

PGS showing obstructive apnoea with hypopnea.

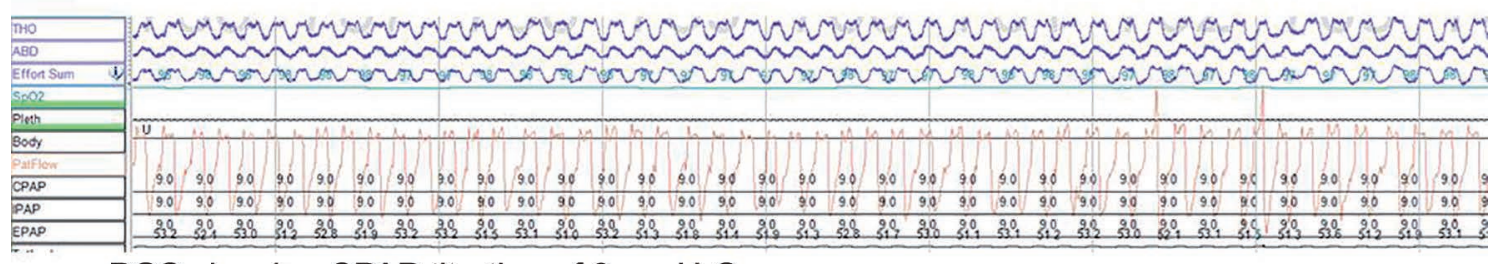

PGS showing CPAP titration of $9 \mathrm{~cm} \mathrm{H} \mathrm{H}_{2} \mathrm{O}$.

Figure 1. First polysomnography showing obstructive apnoea with hypopnea with CPAP titration of $9 \mathrm{~cm} \mathrm{H2O.}$ 


\section{Conclusions}

It is evident that the literature is inadequate to establish the presence of a close association between KLS and sleep apnoea. However, there are reports for which the PSG recordings in KLS do raise possibility of association. Importance of history taking cannot be undermined as diagnosis is limited to history. PSG and SPECT have nothing more to offer apart from being research tool. Possibility of KLS should be considered in cases of OSA with variable response to CPAP.

\section{References}

1. Critchley M, Hoffman HL. The syndrome of periodic somnolence and morbid hunger (Kleine-Levin syndrome). Brit Med J 1942;1:137-9.

2. Gadoth N, Oksenberg A. Kleine-Levin syndrome: an update and mini-review. Brain Dev 2017;39:665-7.

3. Huang Y, Lin Y, Guilleminault C. Polysomnography in KleineLevin syndrome. Neurology 2008;70:795-801.
4. Billiard M, Jaussent I, Dauvilliers Y, Besset A. Recurrent hypersomnia: a review of 339 cases. Sleep Med 2011;15: 247-57.

5. Arnulf I, Zeitzer JM, File J, et al. Kleine-Levin syndrome: a systematic review of 186 cases in the literature. Brain 2005; 128:2763-76.

6. Reimão R, Shimizu MH. Kleine-Levin syndrome. Clinical course, polysomnography and multiple sleep latency test. Case report. Arq Neuropsiquiatr 1998;56:650-4.

7. Luo YW, Yu H, Yuan LH, Zhu GX. A polysomnography study of Kleine-Levin syndrome in a single center. Chin Med J (Engl) 2016;129:1565-8.

8. de Araújo Lima TF, da Silva Behrens NS, Lopes E, Pereira D, et al. Kleine-Levin syndrome: a case report. Sleep Sci 2014;7: 122-5.

9. Lavie P, Klein E, Gadoth N, et al. Further observations on sleep abnormalities in Kleine-Levin syndrome: abnormal breathing pattern during sleep. Electroencephalogr Clin Neurophysiol 1981;52:98-101.

10. Vigren P, Engström M, Landtblom AM. SPECT in the KleineLevin syndrome, a possible diagnostic and prognostic aid? Front Neurol 2014;23:178. 